\title{
Can Extremal Black Holes Have Non-Zero Entropy?
}

\author{
Saurya Das, Arundhati Dasgupta and P. Ramadevi * \\ The Institute of Mathematical Sciences, \\ CIT Campus, Madras - 600 113, India.
}

\begin{abstract}
We give several pieces of evidence to show that extremal black holes cannot be obtained as limits of non-extremal black holes. We review arguments in the literature showing that the entropy of extremal black holes is zero, while that of near-extremal ones obey the Bekenstein-Hawking formula. However, from the counting of degeneracy of quantum (BPS) states of string theory the entropy of extremal stringy black holes obeys the area law. An attempt is made to reconcile these arguments.
\end{abstract}

Typeset using REVTEX

*E-Mail: saurya,dasgupta,rama@imsc.ernet.in 


\section{INTRODUCTION}

Black hole thermodynamics has been an active area of research since it was shown that the area of the event horizon of a black hole can be identified (modulo constants) with the 'entropy' of the black hole. The precise relation is [1,2]:

$$
S_{B H}=\frac{A}{4 G}
$$

where $S_{B H}$ stands for the Bekenstein-Hawking entropy, $A$ is the horizon area and $G$ is Newton's constant. Ever since, it has been one of the outstanding problems to attribute $S_{B H}$ to the degeneracy of quantum states of the black hole. There have been several attempts in this direction and recently some significant work has been done in this area [3,5,6].

It may be noted that, if one considers black holes with either charges or angular momentum or both, then the relation (1) is valid only for non-extremal black holes. However, extremal black holes occupy a special place in black hole physics. They have $T_{H}=0$, ensuring stability against Hawking radiation. Several derivations have been given which demonstrate semi-classically that the entropy of extremal Reissner-Nordström black holes is actually zero [7]. This means that there is a unique quantum microstate corresponding to these black holes. Thus, extremal black holes cannot be regarded as limits of non-extremal black holes because of a discontinuity at that limit. The underlying reason is that nonextremal and extremal black holes are topologically different objects and one cannot change the topology continuously.

On the other hand, string theory predicts that the entropy of extremal black holes follows the relation (1), by suitably identifying these black holes with elementary and/or solitonic string states at weak string coupling [3, 5, 6]. Counting the degeneracy of these states and taking its logarithm reproduces $A / 4 G$ exactly . Thus we are faced with the question : what is the entropy of an extremal black hole? While the proofs given in [7,9] seem quite

\footnotetext{
${ }^{1}$ In certain cases $A$ might correspond to the area of the 'stretched horizon' [3, 4 ].
} 
robust, they are nevertheless semi-classical. Can string theoretic corrections near the horizon modify their result to produce $S=A / 4 G$ ? We address this question in this paper and pose a possible way of resolving this problem.

We first show in section $\mathbb{1}$ as to why the entropy of extremal Reissner-Nordström black holes cannot be given by (四). Next, we summarize the arguments leading to the result that extremal Reissner-Nordström black holes have zero entropy [7,9]. This implies that there is a discontinuous change in the $\mathrm{BH}$ entropy at the extremal limit. We investigate this further in section III to check whether a non-extremal black hole can be transformed into an extremal one by any physical process. In particular, we consider Hawking radiation, Penrose process and super-radiance, by which black holes can lose both mass and charge. In Penrose process and superradiance particles scattering from non-extremal black holes carry away charge and mass from the latter. We show that these processes cannot transform the black hole into an extremal one. Next, considering a non-extremal black hole as a perfect blackbody, radiating with a characteristic Hawking temperature $T_{H}$, we show that the time taken for it to radiate and reach extremality is infinitely large. In other words, the black hole never becomes an extremal one by Hawking radiation. These evidences seem to confirm that extremal black holes are physically quite different from non-extremal ones. In section $\mathbb{\mathbb { }}$, we examine the extremal stringy black holes considered recently in the context of $\mathrm{BH}$ entropy. Following the reasoning of section $\mathbb{1}$ the entropy of these black holes must be zero. However, we propose a modification of the metric and show that it corresponds to the extremal limit of the stringy black hole for which the area law is valid.

\section{ENTROPY OF EXTREMAL BLACK HOLES}

Let us consider the four dimensional Reissner-Nordström (RN) metric;

$$
d s^{2}=-\left(1-\frac{2 G M}{r}+\frac{G Q^{2}}{r^{2}}\right) d t^{2}+\left(1-\frac{2 G M}{r}+\frac{G Q^{2}}{r^{2}}\right)^{-1} d r^{2}+r^{2} d \Omega^{2} .
$$

The following relation holds when the black hole undergoes an infinitesimal changes in mass, charge and horizon area : 


$$
d M=\frac{k}{8 \pi G} d A+\phi d Q
$$

where $M$ and $Q$ are the mass and the charge of the black hole respectively. The horizons are at $r_{ \pm}=G M \pm G \sqrt{M^{2}-Q^{2} / G}$. The surface gravity $\kappa$ is given by $\left(r_{+}-r_{-}\right) / 2 r_{+}^{2}$ and $\phi=Q / r_{+}$is the electrostatic scalar potential at the outer (event) horizon. The Hawking temperature of the black hole is given by $T_{H}=\kappa / 2 \pi$ and $M$ is just the energy $E$ of the black hole. Comparing with the first law of thermodynamics,

$$
d E=T d S-P d V
$$

and replacing $P d V$ by $-\phi d Q$, we see that the entropy $S$ must be identified with $A / 4 G$ \&, thus giving Eq.(11) [2].

Now, for extremal RN black holes, $T_{H}=0$, since $r_{+}=r_{-}$. Thus, we can no longer compare equations (3) and (4) to obtain (1). Moreover, using the relation for temperature

$$
T_{H}^{-1}=\left(\frac{\partial S_{B H}}{\partial M}\right)_{Q},
$$

we see that the right hand side diverges at $T_{H}=0$, indicating that the entropy as a function of $M$ has a singularity at the extremal limit $M=Q / \sqrt{G}$. The above arguments hold good for $D$-dimensional charged black holes as well, for which $T_{H}=(D-3)\left(r_{+}^{D-3}-r_{-}^{D-3}\right) / 4 \pi r_{+}^{D-2}$.

Next, we analyze an alternative derivation of Eq.(1) given in [1], for the case of extremal RN black hole. The entropy is assumed to be an arbitrary function of area:

$$
S_{B H}=f(A)
$$

from which, one can write

$$
\Delta S_{B H}=\frac{d f}{d A} \Delta A
$$

where $\Delta S_{B H}$ and $\Delta A$ correspond to the change in the entropy and area respectively of the black hole when a particle falls into it. The quantity $\Delta S$ can be found from the point of

\footnotetext{
${ }^{2}$ upto an additive constant, which we set to zero by demanding that $S_{B H} \rightarrow 0$ as $M \rightarrow 0$
} 
view of information theory. Before it enters the event horizon, it is certain that the particle exists. Once it enters the horizon, we have no information whatsoever about the particle, and it is justified to assume that it is equally probable for it to exist or not. Thus the minimum entropy change (ignoring possible internal structures of the particle) is given by

$$
(\Delta S)_{\min }=\sum_{n} p_{n} \ln p_{n}=\ln 2
$$

where summation over $n$ corresponds to all possible states of the particle. Now, for an infalling particle with mass $\mu$ and its center of mass at $r_{+}+\delta$, the proper radius $b$ is $\int_{r_{+}}^{r_{+}+\delta} \sqrt{g_{r r}} d r$. Then the minimum change of black hole area accompanied by the absorption of the particle will be [1]

$$
\begin{array}{r}
(\Delta A)_{\min }=2 \mu b, \quad \text { with } \\
b=2 \delta^{1 / 2} \frac{r_{+}}{\sqrt{r_{+}-r_{-}}} .
\end{array}
$$

where $b$ is obtained using $g_{r r}=\left(r-r_{-}\right)\left(r-r_{+}\right) / r^{2}$, and non-extremality condition $\left(r_{+}-r_{-} \gg\right.$ $\delta)$. However, in the extremal limit $\left(r_{+} \rightarrow r_{-}\right)$, we get:

$$
b=\delta+\left.r_{+} \ln \left(r-r_{+}\right)\right|_{r_{+}} ^{r_{+}+\delta}
$$

which diverges for any $\delta>0$. This means that for any finite $\delta$, however small, the corresponding proper radius of the infalling particle is infinite. Thus, the above equation makes sense only for $\delta=0$. Thus, we take $b=0$ corresponding to a point particle resulting in $(\Delta A)_{\min }=0$. Thus to satisfy equation (7) we require,

$$
\left(\frac{\partial f}{\partial A}\right)_{r_{+}=r_{-}} \longrightarrow \infty
$$

which once again shows that the entropy is not continuous at the extremal limit.

The discontinuous nature of entropy under the transition from non-extremal to extremal black hole asserts that the entropy of extremal black holes cannot be determined as a limit of the non-extremal one. Independent derivations of the entropy for extremal and nonextremal black holes have been given [9] which are in conformity with the above result. It 
has been shown that the topology of the black hole near the horizon plays a crucial role in determining the entropy. We now briefly review these arguments.

The Euclideanised metric in $d$ dimensions near the horizon is

$$
d s^{2}=N^{2} d \tau^{2}+N^{-2} d r^{2}+r^{2} d \Omega_{d-2}^{2}
$$

For the above metric, the proper angle $\Theta$ in the $r-\tau$ plane near the horizon is defined as

$$
\Theta \equiv \frac{\text { proper length }}{\text { proper radius }}=\frac{\int_{t_{1}}^{t_{2}} \sqrt{g_{\tau \tau}} d \tau}{\int_{r_{+}}^{r} \sqrt{g_{r r}} d r}=\left.\left(N N^{\prime}\right)\right|_{r_{+}}\left(t_{2}-t_{1}\right)
$$

where the prime denotes differentiation with respect to $r$. It can be shown that $N$ satisfies the following relation:

$$
\left(t_{2}-t_{1}\right) N^{2}=2 \Theta\left(r-r_{+}\right)+O\left[\left(r-r_{+}\right)^{2}\right]
$$

Also, the two dimensional metric near the horizon can be written in the form

$$
d s^{2}=d \rho^{2}+\rho^{2} d \Theta^{2}
$$

where $\rho \equiv \sqrt{2\left(r-r_{+}\right) / N N^{\prime}}$. To avoid a conical singularity at the horizon, the period of $\Theta$ is identified with $2 \pi$, which corresponds to the topology of a disc with zero deficit angle in the $r-\tau$ plane. This can always be done for non-extremal black holes, as $\left.\left(N N^{\prime}\right)\right|_{r_{+}}$in Eq.(倩) is non-zero. However, for extremal black holes, the proper radius diverges (see Eq.(10)), and hence the proper angle tends to zero. Thus the conical deficit angle becomes $2 \pi$ and the topology is that of an annulus [9]. The topology of the transverse section in either case is $S^{d-2}$.

Now, we need to see how this topology reflects on the entropy calculation. Treating the black holes as microcanonical ensemble, the action $I$ in the Hamiltonian formulation of gravity is proportional to entropy. The dimensional continuation of Gauss-Bonnet theorem to $d$ dimensions [9, 10] determines

$$
I \propto \chi A_{d-2}
$$


where $\chi$ is the euler characteristic of the Euclideanised $r-\tau$ plane and $A_{d-2}$ is the area of the transverse $S^{d-2}$. The exact expression for the black hole entropy is given by

$$
S=\frac{\chi A}{4 G}
$$

For non-extremal black holes $\chi=1$ (disc), leading to the area law (11), while for extremal black holes $\chi=0$ (annulus), implying a vanishing entropy.

It has also been argued by Hawking et al [7,8], that the Euclidean action for extremal black holes is proportional to the inverse Hawking temperature $(\beta)$ in a canonical ensemble

leading to the vanishing entropy. This follows from the relations $S=-\left(\beta \frac{\partial}{\partial \beta}-1\right) \ln Z$, and $Z=e^{-I}$. P

Thus, it is clear that extremal black holes cannot be thought of as limits of non-extremal black holes at least as far as the expression for their entropies are concerned. In the next section, we investigate some physical processes which further support this conclusion.

\section{PHYSICAL PROCESSES}

For the charged non-extremal black holes, we know that Hawking radiation is dominant in the energy regime $\omega>e \phi$ where $e$ is the charge of the emitted particles. On the other hand, the Penrose process (and its quantum analog - superradiance) is significant when $\omega<e \phi$. We study both the processes, thus spanning all the energy regimes, to confirm that non-extremal black holes cannot transform into extremal ones.

\section{A. Superradiance and extremality}

RN black holes can lose mass and charge by processes like Penrose process and superradiance, which are dominant for low energies of the infalling particles. We examine here, whether a non-extremal black hole can reach extremality through these processes.

\footnotetext{
${ }^{3}$ Extremal black hole entropy has been explored by alternative methods as well in [1].
} 
The energy of a particle in a $4 D \mathrm{RN}$ background is given by 12

$$
E=m \sqrt{\left(1-\frac{r_{+}}{r}\right)\left(1-\frac{r_{-}}{r}\right)}+\frac{e Q}{r} .
$$

Where $m$ and $e$ are the mass and charge of the infalling particle. If this particle has a charge opposite to that of the black hole, then sufficiently close to the horizon, the first term tends to zero, making the energy negative. Hence in this regime,

$$
|E|<\frac{|e Q|}{r_{+}}
$$

If two oppositely charged bound particles with total energy $E_{0}$ fall near the black hole and separate there, one of the charges can have negative energy by the above argument. The particle with negative energy will fall into the black hole and the other particle escapes. By conservation of energy,

$$
\begin{aligned}
& E_{2}^{\prime}=E_{0}+\left|E_{1}\right| \\
& M^{\prime}=M-\left|E_{1}\right|
\end{aligned}
$$

$E_{2}^{\prime}$ is the energy of the particle which escapes, $E_{1}$ is the energy of the particle which falls into the black hole and $M^{\prime}$ is the final mass of the black hole. Thus there is a decrease in mass of the black hole, while the escaping particle carries back more energy. Also as an oppositely charged particle is absorbed by the black hole, it's effective charge decreases to become $Q^{\prime}=Q-e$ Since for a non-extremal black hole $\left(\sqrt{G} Q / r_{+}<1\right)$. We find from equation(19), that

$$
\frac{e}{\sqrt{G}}>\left|E_{1}\right|
$$

In other words the decrease of mass of the black hole will be less than the decrease of charge due to this process. Hence, the condition of non-extremality $M>Q / \sqrt{G}$ will be maintained as the rate of charge loss will exceed the rate of mass loss.

The quantum analog for this phenomenon is Superradiance. Fields with low energy are shown to be scattered away from the black hole such that the reflection coefficient is greater 
than one. The charged scalar field equation can be solved in the RN back ground and the following relation for the reflection coefficient $|R|^{2}$ and transmission coefficient or the absorption coefficient $|T|^{2}$, can be obtained as [15],

$$
1-|R|^{2}=\frac{1}{k}\left(\omega-\frac{e Q}{r_{+}}\right)|T|^{2}
$$

For $\omega<e Q / r_{+}$, the reflection coefficient, $|R|^{2}$ is greater than 1 , or the scalar wave takes away energy from the black hole. The condition for superradiance is thus

$$
m<\omega<\frac{e Q}{r_{+}}
$$

The rate of charge loss and mass loss for the black hole is

$$
\begin{aligned}
\frac{d Q}{d t} & =-e \int_{m}^{\frac{e Q}{r_{+}}}|R|^{2} d \omega \\
\frac{d M}{d t} & =-\int_{m}^{\frac{e Q}{r_{+}}}|R|^{2} \omega d \omega .
\end{aligned}
$$

We find that the for the initial value of the integrands, $(e / \sqrt{G})|R(m)|^{2}>m|R(m)|^{2}$. Thus as equation (24) holds for each value of $\omega$, which is bounded from above,

$$
\left|\frac{d Q}{d t}\right|>\sqrt{G}\left|\frac{d M}{d t}\right|
$$

Hence from the quantum process also it is clear that the $M>Q / \sqrt{G}$ condition will be maintained.

The above result is easily extendible to higher dimensional charged dilatonic black holes. The equation for classical energy of a charged particle in a generic charged black hole back ground is

$$
E=m \sqrt{\left(1-\left(\frac{r_{+}}{r}\right)^{D-3}\right)\left(1-\left(\frac{r_{-}}{r}\right)^{D-3}\right)^{1-2 a^{2} /\left(D-3+a^{2}\right)}}+\frac{e Q}{r^{D-3}} .
$$

Here $D$ is the dimension of space and $a$ stands for a parameter which interpolates between the general relativistic solution $a=0$ and the dilatonic stringy black hole $a=1$. As

\footnotetext{
${ }^{4}$ for the $D$-dimensional charged metric, see 17].
} 
$r_{+}^{D-3}=G M+G \sqrt{M^{2}-Q^{2} / G}$, non-extremality will imply $\sqrt{G} Q<r_{+}^{D-3}$ and equation (22), hold for these. The equation for the reflection and transmission coefficients for these black holes has been calculated in [16]. The condition for superradiance equation (24), is the same for these black holes.

Apart from the induced process stated above, the black hole loses charge spontaneously by vacuum polarization as shown in [15]. For this the rate of charge loss will also be very high, and the black hole will tend to discharge itself very fast. The $M=Q / \sqrt{G}$ condition, once again, will not be obtained. Thus in the processes considered so far, the extremality condition cannot be attained from a non-extremal state.

\section{B. Hawking Radiation and Extremality}

In this section, we consider mass and charge loss of black holes by Hawking radiation. When the RN black hole radiates, the spectrum of particles is given by the Planck distribution [2]:

$$
d E_{\omega}=\frac{(\omega-e \phi)^{3} d \omega}{e^{(\omega-e \phi) / T_{H}}-1}
$$

where $d E_{\omega}$ is the radiation energy in the spectral range $\omega$ to $\omega+d \omega$. Integrating over $\omega$ from $e \phi$ to $\infty$, one obtains the rate at which the black hole loses energy, i.e. mass 12

$$
\frac{d M}{d t}=-\sigma T_{H}^{4} A
$$

$A=4 \pi r_{+}^{2}$ being the area of the event horizon and $\sigma$ the Stefan-Boltzmann constant. Thus, for RN black holes with $T_{H}=\left(r_{+}-r_{-}\right) / 4 \pi r_{+}^{2}$, it is given by,

$$
\frac{d M}{d t}=-\frac{\sigma}{(4 \pi)^{3}} \frac{\left(r_{+}-r_{-}\right)^{4}}{r_{+}^{6}}
$$

We integrate (31) to get,

$$
\int_{0}^{t_{0}} d t=-\frac{(4 \pi)^{3}}{\sigma} \int_{M_{0}, Q_{0}}^{M^{\prime}, Q^{\prime}} \frac{r_{+}^{6} d M}{\left(r_{+}-r_{-}\right)^{4}} .
$$


Here $t_{0}$ is the time taken for the black hole to reach a final state with mass and charge $M^{\prime}$ and $Q^{\prime}$ respectively, from their initial values $M_{0}$ and $Q_{0}$. We are interested in calculating $t_{0}$ to reach a final extremal state (i.e. $M^{\prime}=Q^{\prime} / \sqrt{G}$ ). from a non-extremal initial state. For simplicity, let us first assume that the radiated particles are electrically neutral, i.e. $e=0$ and hence $Q$ is a constant. Then, the time taken for the black hole to become extremal is:

$$
\begin{aligned}
\int_{0}^{t_{0}} d t & =-\frac{(4 \pi)^{3}}{\sigma} \lim _{M^{\prime} \rightarrow Q_{0} / \sqrt{G}} \int_{M_{0}}^{M^{\prime}} \frac{r_{+}^{6} d M}{\left(r_{+}-r_{-}\right)^{4}}, \\
& =\frac{(4 \pi)^{3}}{\sigma} \lim _{M^{\prime} \rightarrow Q_{0} / \sqrt{G}}\left[\frac{105}{12} Q_{0}^{3} \sqrt{G} \ln \left(\frac{M+Q_{0} / \sqrt{G}}{M-Q_{0} / \sqrt{G}}\right)\right. \\
& \left.+\left(\frac{328 Q_{0}^{4}-128 M^{4} G^{2}-128 Q_{0}^{2} M^{2} G}{12\left(M^{2}-Q_{0}^{2} / G\right)^{1 / 2}}\right)+\left(\frac{198 Q_{0}^{4} M-128 M^{5} G^{2}-64 Q_{0}^{2} M^{3} G}{12\left(M^{2}-Q_{0}^{2} / G\right)}\right)\right]\left.\right|_{M=M_{0}} ^{M=M^{\prime}}
\end{aligned}
$$

Clearly, $t_{0}$ diverges. That is, the RN black hole which emits neutral particles, takes an infinite amount of time to reach extremality. Generalizing the proof for Hawking particles carrying charges is not difficult. Then $Q$ is not a constant in Eq.(32). However, as before, the integrand on the right hand side diverges as $r_{+} \rightarrow r_{-}$and thus $t_{0} \rightarrow \infty$. Identical conclusions follow for general relativistic charged black holes in $D$-dimensions, for which, the rate of mass loss is given by

$$
\frac{d M}{d t}=-\sigma_{D} A_{D-2}\left(\frac{D-3}{4 \pi}\right)^{D} \frac{\left[r_{+}^{D-3}-r_{-}^{D-3}\right]^{D}}{r_{+}^{(D-2)(D-1)}},
$$

$\sigma_{D}$ being the $D$-dimensional Stefan-Boltzmann constant and $A_{D-2}$ the area of unit $S^{D-2}$. Here too the integrand diverges in the extremal limit. In general, $t_{0} \rightarrow \infty$ whenever $T_{H}=0$ for the extremal black hole. We shall see later, that this includes a certain class of stringy black holes.

Thus, we conclude that a extremal black hole state with $T_{H}=0$ cannot be reached in a finite time by Hawking radiation from a non-extremal black hole. This is in conformity with the third law of black hole thermodynamics, which asserts that the same cannot be reached in a finite sequence of operations [13]. These also provide pieces of evidence that the area law for the entropy of non-extremal black holes cannot be extended to the case of extremal 
black holes. In the next section, we will study the entropy of certain extremal stringy black holes which supposedly obey the area law.

\section{EXTREMAL BLACK HOLES IN STRING THEORY}

The degeneracy counting of string states saturating the BPS bound has been claimed to give the entropy of the extremal stringy black holes with the same mass and charge. The surprising fact is that the entropy obtained by degeneracy counting matches the area law, which is applicable only to non-extremal black holes. We will try to reconcile this apparent discrepancy in this section.

There are two types of extremal stringy black holes which saturate the BPS bound :

1. The horizon merges with the curvature singularity. These black holes have zero horizon area and the dilaton field becomes singular at the horizon.

2. The two event horizons coincide as in General relativity. The area for these black holes is non-zero and the dilaton is regular at the horizon.

A few examples of these extremal stringy black holes and their properties are tabulated below.

\begin{tabular}{||c|c|c|c|c|c|c|c||}
\hline \multirow{2}{*}{ Type } & \multirow{2}{*}{ Example } & \multicolumn{2}{|c|}{$T_{H}$} & \multicolumn{2}{c|}{ Macro Entropy } & \multicolumn{2}{|c||}{ Micro Entropy } \\
\cline { 3 - 8 } & & $\mathrm{NE}$ & $\mathrm{E}$ & $\mathrm{NE}$ & $\mathrm{E}$ & $\mathrm{NE}$ & $\mathrm{E}$ \\
\hline 1 & Het. on $T^{6}$ & $\neq 0$ & $1 / 4 \pi m_{0}$ & $A / 4$ & 0 & - & $A_{s t} / 4$ \\
\hline 2 & II B on $K^{3} \times S^{1}$ & $\neq 0$ & 0 & $A / 4$ & 0 & - & $A / 4$ \\
\hline
\end{tabular}

where $\mathrm{NE}=$ nonextremal, $\mathrm{E}=$ extremal and $A_{s t}$ is the area of the stretched horizon. The examples referred to here are taken from Refs. [3,5].

The first type of extremal black holes [3], obtained by compactifying heterotic on $T^{6}$ has $T_{H} \neq 0$. Hence in accordance with the third law of black hole thermodynamics, the extremal 
state can be reached in a finite sequence of steps. In particular, one can see from Section IIIB, that the time $t_{0}$ to reach extremality by Hawking radiation is finite f. Similarly, the discussions in Section [1] based on [1,2] is valid for this example since proper radius is finite. It follows that these extremal black holes can be regarded as limits of non extremal ones and their entropy obeys the area law. In order to determine this entropy, we look at the extremal stringy black hole solution of the low energy effective action of heterotic string theory compactified on $T^{6}$. The Euclideanised metric near the horizon is [3]:

$$
d \bar{s}^{2}=\frac{1}{4} \bar{r}^{2} d \bar{\tau}^{2}+d \bar{r}^{2}+\frac{1}{4} \bar{r}^{2}\left(d \theta^{2}+\sin ^{2} \theta d \phi^{2}\right)
$$

and the solution for the dilaton field is $e^{\bar{\phi}}=\bar{r}^{2} / 4$. Here $\bar{r}^{2}=4 g r$ and $\bar{\tau}=\tau / m_{0}$ where $g$ is the string coupling and the parameter $m_{0}$ is related to the mass of the black hole. Note that the topology near the horizon $(\bar{r}=0)$ is disc $\times S^{2}$, which is that of a generic non-extremal black hole. Although $S=A / 4 G$, the entropy vanishes as the horizon area is zero. On the other hand, the degeneracy counting of the elementary BPS string states gives a non-zero result. It has been proposed that stringy corrections near the horizon modifies the metric and the dilaton such that the results agree. A possible modification of the metric and the dilaton is

$$
d \bar{s}^{2}=\frac{1}{4} \bar{r}^{2} d \bar{\tau}^{2}+d \bar{r}^{2}+\frac{1}{4} f_{1}(\bar{r})\left(d \theta^{2}+\sin ^{2} \theta d \phi^{2}\right) \quad ; \quad e^{\phi}=f_{2}(\bar{r})
$$

where $f_{1}(\bar{r})$ and $f_{2}(\bar{r})$ are two smoothing functions which are positive constants at $\bar{r}=0$ and equal $\bar{r}^{2} / 4$ for large $\bar{r}$. Now, the horizon area can be shown to be $\sqrt{m_{0} f_{1}(0) / g}$, which is finite and proportional to the logarithm of the number of corresponding elementary string states satisfying the BPS bound. Here $f_{1}(0)$ is obtained by a fit with the degeneracy.

\footnotetext{
${ }^{5}$ The grey-body factor for the metric (34), for low energy quanta, seems to be zero because of vanishing horizon area. However, as argued in [3], this metric suffers large stringy corrections resulting in the metric (35) which has a non-zero (stretched) horizon area.
} 
The second type of the stringy extremal black holes obtained from type IIB string theory compactified on $K^{3} \times S^{1}$, on the contrary, has $T_{H}=0$. The horizon area $A \neq 0$ if both NSNS charge $Q_{H}$ and R-R charge $Q_{F}$, associated with the $\tilde{H}$ and $F$ field strengths respectively, are non-zero [5]. The extremal black hole solution from low energy effective theory is the five-dimensional RN metric [17]:

$$
d s^{2}=-\left(1-\left(\frac{r_{0}}{r}\right)^{2}\right)^{2} d t^{2}+\left(1-\left(\frac{r_{0}}{r}\right)^{2}\right)^{-2} d r^{2}+r^{2} d \Omega_{3}^{2} .
$$

where the horizon radius in terms of the charges $Q_{H}$ and $Q_{F}$ is $r_{0}=\left(8 Q_{H} Q_{F}^{2} / \pi^{2}\right)^{1 / 6}$. As discussed in Section [1], the Euclidean topology is annulus $\times S^{3}$ and hence its entropy is zero. The degeneracy counting has been done by identifying a collection of BPS saturated D-brane states in weak coupling regime with the extremal charged black holes at strong coupling. The logarithm of this degeneracy exactly matches the area law for the metric (36). The method was applied to certain four dimensional stringy black holes as well, with identical conclusions [18].

The previous discussions suggest that there could be string theoretic or other quantum gravity corrections which would prevent the metric near the horizon from being exactly extremal, such that the area law continues to be valid. It has been argued that Planck scale effects become important near the horizon [19,20]. Stringy modifications were also anticipated in [6] on the basis of stability requirements. In view of the above, the modified metric with the correct topology should be of the form,

$$
d s^{2}=-f(r)\left(1-\left(\frac{r_{0}}{r}\right)^{2}\right) d t^{2}+f(r)^{-1}\left(1-\left(\frac{r_{0}}{r}\right)^{2}\right)^{-1} d r^{2}+r^{2} d \Omega_{3}^{2},
$$

where $f(r)$ is a positive definite and bounded function of $r$ in the range $r_{0} \leq r<\infty$, such that $f\left(r_{0}\right) \neq 0$, although it can be arbitrarily small. The corresponding Hawking temperature gets modified from zero to $T_{H}=f\left(r_{0}\right) / 2 \pi r_{0}$.

However though the exact nature of the stringy corrections are not ascertained, the metric (37) can be understood from an alternative approach. In general the metric solution of type IIB action compactified on five dimensional manifold is of the form [21]: 


$$
d s^{2}=-f^{-2 / 3}\left(1-\frac{a^{2}}{\bar{r}^{2}}\right)+f^{1 / 3}\left[\left(1-\frac{a^{2}}{\bar{r}^{2}}\right)^{-1} d \bar{r}^{2}+r^{2} d \Omega_{3}^{2}\right]
$$

where,

$$
f=\left(1+\frac{a^{2} \sinh ^{2} \alpha}{\bar{r}^{2}}\right)\left(1+\frac{a^{2} \sinh ^{2} \gamma}{\bar{r}^{2}}\right)\left(1+\frac{a^{2} \sinh ^{2} \sigma}{\bar{r}^{2}}\right)
$$

Here, $\alpha, \gamma$ and $\sigma$ are three boost parameters. These parameters along with $a$, radius $R$ of $S^{1}$ and volume $V$ of the four dimensional compact manifold determine the number of one branes, five branes, the corresponding anti-branes and the momentum in the $S^{1}$ direction. The five dimensional Reissner-Nordstörm metric is obtained when all these boost parameters are equal, i.e. $\alpha=\gamma=\sigma$, and simultaneously doing the coordinate transformation $r^{2}=\bar{r}^{2}+$ $a^{2} \sinh ^{2} \alpha:$

$$
\begin{aligned}
d s^{2} & =-\left[1-\frac{\left(a \sinh ^{2} \alpha\right)^{2}}{r^{2}}\right]\left[1-\frac{\left(a \cosh ^{2} \alpha\right)^{2}}{r^{2}}\right] d t^{2}+\frac{d r^{2}}{\left[1-\frac{\left(a \sinh ^{2} \alpha\right)^{2}}{r^{2}}\right]\left[1-\frac{\left(a \cosh ^{2} \alpha\right)^{2}}{r^{2}}\right]} \\
& +r^{2} d \Omega_{3}^{2} .
\end{aligned}
$$

The two horizons are at

$$
r_{+}=a \cosh \alpha, \quad r_{-}=a \sinh \alpha
$$

It is evident that the extremal limit $\left(r_{+} \rightarrow r_{-}\right)$is achieved when the boost parameter becomes indefinitely large, i.e. $\alpha \rightarrow \infty$, and $a \rightarrow 0$ such that $a e^{\alpha}$ is held fixed. Clearly, taking this limit does not change the Euclidean topology from disc $\times S^{3}$ to annulus $\times S^{3}$ as exact equality of the horizons is not achieved. The metric is of the proposed form (37), coinciding with the metric (36) only in a limiting way. Therefore the black hole metric considered in [5] obeys the area law for entropy. The stability of the black hole is ensured by the fact that the Hawking temperature, $T_{H}=1 / 2 \pi a \cosh ^{3} \alpha$, is infinitesimal.

¿From the point of view of counting microscopic degrees of freedom using D-brane techniques, it has been shown that the density of states varies continuously as a function of parameters 22, 23]. In other words, there is no discontinuity at the BPS limit and the entropy calculated from the degeneracy of BPS states is to be identified with $A / 4 G$ of the corresponding black hole in the extremal limit. 


\section{CONCLUSIONS}

In this paper, we have shown that non-extremal and extremal black holes are physically quite distinct objects and it is impossible to transform the former to the latter by physical processes. These have different Euclidean topologies and hence they do not share the same entropy formula. Extremal black holes have zero entropy as opposed to non-extremal black holes which obey the area law. Since the degeneracy counting for BPS saturated states follows the area law, we have proposed a form for the black hole metric which has the above property. We have justified this proposal by showing that this metric corresponds to the extremal limit of the black hole solutions in string theory. Finally, we have pointed out that the BPS saturated D-brane configuration should actually be identified with the corresponding black hole in the extremal limit (as opposed to exactly extremal) such that the area law for entropy is valid. The interesting question remains as to what kind of statistical interpretation can be given to the exactly extremal black holes and whether they have a stringy interpretation. We hope to report on it elsewhere.

\section{ACKNOWLEDGEMENTS}

We thank P. Majumdar for discussions and for carefully reading the manuscript and suggesting various improvements. We thank T. Jayaraman, T. Sarkar and G. Sengupta for discussions. We are grateful to A. Sen for useful correspondence. One of us (A.D.) would like to thank F. Englert for discussions. 


\section{REFERENCES}

[1] J. Bekenstein, Phys. Rev. D7, 2333 (1973).

[2] S. W. Hawking, Phys. Rev. D13, 191 (1976).

[3] A. Sen, Mod. Phys. Lett. A10, 2081 (1995) (hep-th/9504147).

[4] A. Peet, Nucl. Phys. B (to be published) (hep-th/9506200).

[5] A. Strominger and C. Vafa, Phys. Lett. B379, 99 (1996) (hep-th/9601029).

[6] G. T. Horowitz, The Origin of Black Hole Entropy in String Theory, gr-qc/9604051.

[7] S. W. Hawking, G.T. Horowitz and S. F. Ross, Phys. Rev. D51, 4302 (1995) (grqc/9409013).

[8] S. W. Hawking and G. T. Horowitz, Class. Quantum. Grav. 13, 1487 (1996).

[9] C. Teitelboim, Phys. Rev. D51, 4315 (1995) (hep-th/9410103).

[10] M. Banados, C. Teitelboim and J. Zanelli, Phys. Rev. Lett. 72, 957 (1994).

[11] A. Ghosh and P. Mitra, Phys. Lett. 357, 295 (1995) (hep-th/9411128).

[12] R. M. Wald, General Relativity, University of Chicago Press (Chicago and London), 1984.

[13] J. M. Bardeen, B. Carter and S. W. Hawking, Com. Math. phys. 31, 161 (1973).

[14] D. Christodoulou and R. Ruffini, Phys. Rev. D4, 3552 (1971).

[15] G. W. Gibbons, Com. Math. Phys. 44, 245 (1975).

[16] K. Shiraishi, Mod. Phys. Lett. 7, 3449 (1992).

[17] G. W. Gibbons and K. Maeda, Nucl. Phys. B298, 741 (1988).

[18] J. M. Maldacena and A. Strominger, Phys. Rev. Lett. 77, 428 (1996) (hep-th/9603060);

C. V. Johnson, R.R. Khuri and R.C. Myers, Phys. Lett. B378, 78 (1996) hep- 
th/9603061); A. A. Tseytlin, Extremal black hole entropy from conformal string sigma model, hep-th/9605091; N. Hambli, Phys. Rev. D. (to be published) (hep-th/9605236).

[19] L. Susskind, L. Thorlacius and J. Uglum, Phys. Rev. D48, 3743 (1993) (hepth/9306069).

[20] G. 't Hooft, Scattering Matrix Approach for the Quantum Black Hole, gr-qc/9607022.

[21] G. T. Horowitz, J. M. Maldacena and A. Strominger, Phys. Lett. B383, 151 (1996) (hep-th/9603109).

[22] G. T. Horowitz and A. Strominger, Phys. Rev. Lett. 77, 2368 (1996) hep-th/9602051).

[23] J. Maldacena, D-branes and Near Extremal Black Holes at Low Energies, hepth/9611125. 